\title{
Dependence of Gelation and Fusion Behavior of Poly(vinyl chloride) Plastisols upon Particle Size and Size Distribution
}

\author{
N. NAKAJima, J. D. ISNer, E. R. HARRELl, \\ and C. A. DANIELS \\ BFGoodrich Chemical Group, Technical Center, P.O. Box 122, \\ Avon Lake, Ohio 44012, U.S.A.
}

(Received April 23, 1981)

\begin{abstract}
Poly(vinyl chloride) (PVC) plastisol is a suspension of small particles of PVC resin in plasticizer. The processing of plastisol typically involves flow at room temperature during coating or mold filling, and gelation and fusion as the plastisol is heated to form the final product. This paper explores the effect of the particle size distribution on the gelation and fusion behavior. For this purpose a commercial PVC resin was air-classified into fractions of different particle size. Samples were then reconstituted to have known particle size distributions. Particular emphasis is placed on the effect of the fine particle fraction on the material behavior. The progress of gelation and fusion were followed by measurement of the viscoelastic properties as a function of treatment temperature using a mechanical spectrometer. Characteristic changes in the viscoelastic behavior were associated with changes in morphology, observed with a scanning electron microscope, enabling qualitative discrimination of the gelation and fusion processes. The larger fraction of fine particles gave higher values of $G^{\prime}$ and $G^{\prime \prime}$. It seems that the fine particles promote the interparticulate bonding, when the particles start fusing together.

KEY WORDS Poly(vinyl chloride) / Plastisol / Particle Size Distribution / Gelation-Fusion / Particulate Morphology / Viscoelastic Properties /
\end{abstract}

Both the average particle size and the particle size distribution of poly(vinyl chloride) (PVC) dispersion resins have been recognized as important material variables pertinent to PVC plastisol behavior. The flow behavior at room temperature is profoundly influenced by these variables. Therefore, they are important in regard to processability. The types of flow behavior encountered in these systems, such as thixotropy, shear-thinning and dilatancy have been investigated previously. ${ }^{1}$ The oscillatory measurements were also carried out to characterize the dynamic responses. ${ }^{2}$ On the other hand, less attention was paid to the effect of particle size and size distribution on gelation and fusion. Since gelation and fusion are also important steps in plastisol processing, we have undertaken this study.

Our previous studies demonstrated the effectiveness of viscoelastic measurements for characterizing gelation and fusion behavior. ${ }^{3-5}$ Morphology was also examined of samples taken at different stages of gelation and fusion. ${ }^{5}$ These observations elucidated the mechanism of the diffusion of plasticizer into resin particles, the presence and disappearance of particulate structure and the temperature range of the melting of the PVC crystallites. In addition, the progress of interparticle bonding was postulated to explain the accompanying development of mechanical strength.

When the plasticizer is taken up by the resin particles, a large amount of interparticle voids are present; these voids disappear, of course, when the particulate structure disappears. However, some defects are present up until the fusion is complete; the latter state is reached when the mechanical strength is fully developed.

In the present work a commercial dispersion resin was air-classified. The gelation and fusion behavior of the coarse and fine fractions was examined. The effect of blending these fractions so as to produce known size distribution(s) was also investigated. 


\section{EXPERIMENTAL}

\section{PVC Dispersion Resin Samples}

The samples were the same as those used in our previous study. ${ }^{2}$ The commercial resin, unfractionated, was designated as the raw feed, RF, which was air-classified into five different size fractions. The finest fraction, FF, and the coarsest one, 4C, were used in this report singly or as blends of these two in different proportions.

Table I lists the ingredients used in the plastisol formulation for this study.

Table I. Plastisol formulation

\begin{tabular}{lc}
\hline & Parts by weight \\
\hline PVC Resin & 100 \\
Plasticizer, DOP & \\
$\quad$ (di-2-ethylhexylphthalate) & 60 \\
Epoxydized soybean oil & 5 \\
Barium, cadmium, zinc stabilizer & 3 \\
\hline
\end{tabular}

\section{Particle Size Determination}

Particle size distributions were analyzed with the Micromeritics Sedigraph. ${ }^{6,7}$ All measurements were made to determine particle size distribution as it existed in the plastisol. Therefore, a given plastisol was diluted with dibutyl sebacate with gentle mechanical agitation.

\section{Viscoelastic Properties}

The Rheometrics Mechanical Spectrometer, ${ }^{8}$ RMS, was used with a $50 \mathrm{~mm}$ diameter, parallel plate assembly. The frequency of oscillation was
$0.159 \mathrm{~Hz},\left(1 \mathrm{rad} \mathrm{s}^{-1}\right)$. The heating was a $5^{\circ} \mathrm{C} \mathrm{min}^{-1}$ step-change in temperature, which gave nearequilibrium values for the viscoelastic properties.

\section{Morphology}

The AMR model 1400 Scanning Electron Microscope (SEM) was used. ${ }^{5,9}$ A series of specimens was prepared from a given plastisol, each specimen corresponding to a particular stage (temperature) of gelation and fusion. That is, the molding of these specimens was done in situ during the viscoelastic measurements, which were terminated at different temperatures. The specimens were then freeze-fractured at liquid nitrogen temperature and the fracture surface was examined by SEM.

\section{RESULTS}

\section{Particle Size Distribution}

The particle size distributions of RF, FF and $4 \mathrm{C}$ are shown in Figure 1, where the cumulative massfraction is plotted from larger to smaller size as a function of the logarithm of the corresponding equivalent spherical diameter. The distributions of FF-4C blends are shown in Figure 2. The observed distributions of the blends are different from those calculated from the data of the components. In particular, it was observed that there were fewer larger particles than predicted, indicating that deagglomeration of the resin took place during blending.

Samples RF and 4C have approximately bimodal distributions. The diameter at $50 \%$ mass of the coarse portion was $13-15 \mu \mathrm{m}$ and that of the fine

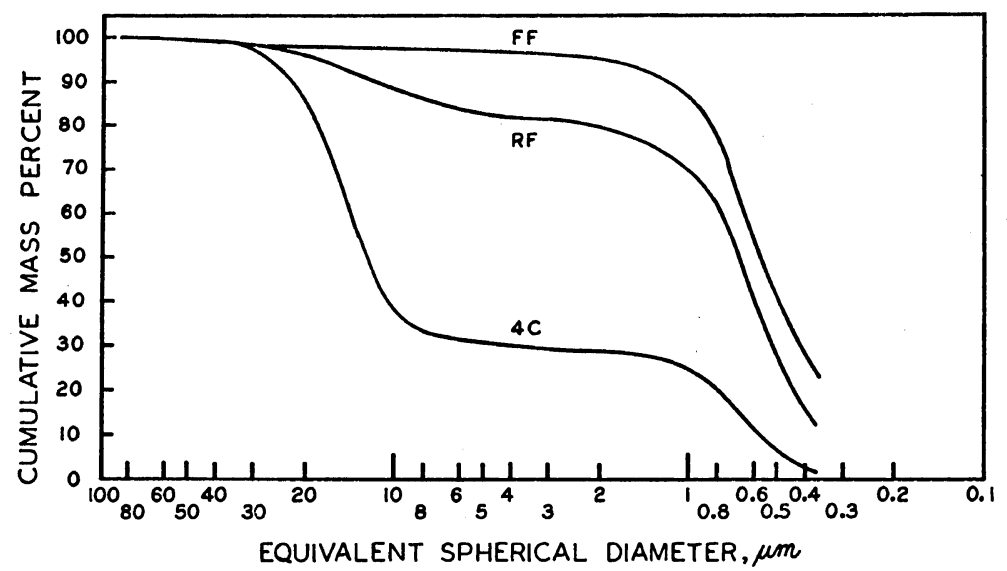

Figure 1. Cumulative particle size distribution of samples RF, FF, and 4C. 


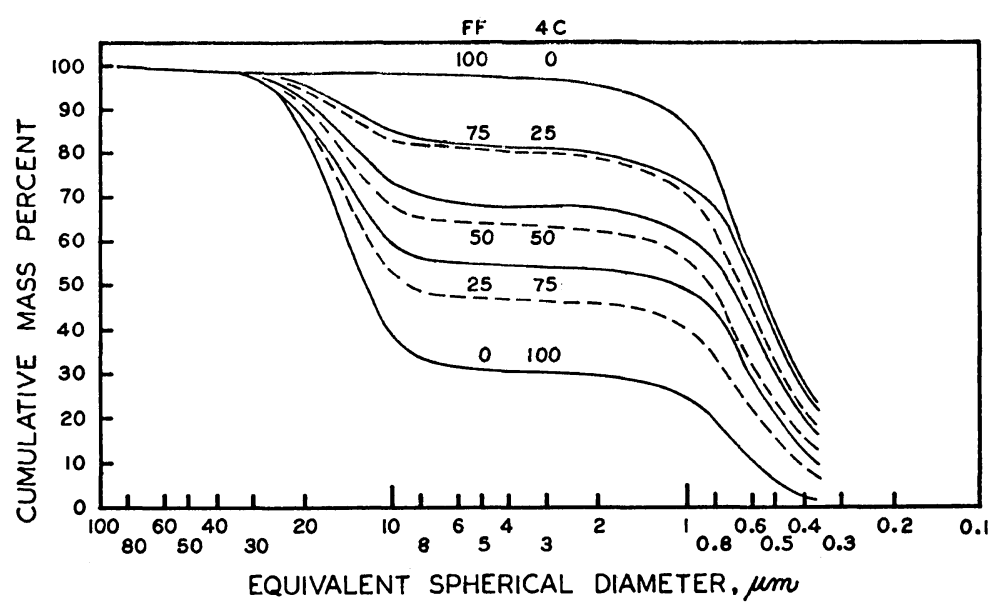

Figure 2. Observed and calculated cumulative particle size distribution of FF-4C blends: - - , observed; -- , calculated.

Table II. Particle size distribution

\begin{tabular}{|c|c|c|c|c|c|}
\hline & \multicolumn{3}{|c|}{ Mass Fraction $/ \%$} & \multicolumn{2}{|c|}{ Median diameter, $\mu \mathrm{m}$} \\
\hline & Coarse & Fine and ultrafine & Ultrafine & Coarse & Fine \\
\hline Sample & $>3.5 \mu \mathrm{m}$ & $\langle 3.5 \mu \mathrm{m}$ & $\langle 0.37 \mu \mathrm{m}$ & & \\
\hline FF & 3 & 97 & 24.5 & $(15)^{\mathrm{a}}$ & 0.66 \\
\hline $4 \mathrm{C}$ & 70 & 30 & 2.0 & 15.1 & 0.73 \\
\hline RF & 18 & 82 & 12.5 & 13.0 & 0.65 \\
\hline \multicolumn{6}{|l|}{$\mathrm{FF}-4 \mathrm{C}$} \\
\hline $75: 25$ & 19 & 81 & 22.5 & 14.2 & 0.58 \\
\hline $50: 50$ & 32 & 68 & 17.0 & 14.8 & 0.60 \\
\hline $25: 75$ & 46 & 54 & 10.0 & 15.1 & 0.62 \\
\hline
\end{tabular}

a Estimated.

portion was $0.60-0.73 \mu \mathrm{m}$. The difference between $4 \mathrm{C}$ and RF was in the proportions of the coarse and fine components. The FF fraction was essentially monomodal and the diameter at 50 cumulative mass percent was $0.66 \mu \mathrm{m}$. The finer particle portion in $\mathrm{FF}, 4 \mathrm{C}$ and $\mathrm{RF}$ is the primary particles formed during polymerization; the coarse particles are the agglomerates formed during spraydrying. The particle size distribution data are also summarized in Table II.

\section{VISCOELASTIC MEASUREMENTS}

Figure 3 shows the viscoelastic profile of the gelation and fusion behavior of the plastisol prepared solely from the FF fraction. The solid and broken lines are the duplicate runs up to $210^{\circ} \mathrm{C}$. The dash-dot line represents a run terminated at $160^{\circ} \mathrm{C}$. The other four markings are runs which terminated at temperatures indicated by the respective markings. The most important observation is that there was considerable difficulty in reproducing data between 81 and $160^{\circ} \mathrm{C}$. These temperatures correspond respectively to the glass transition temperature, $T_{\mathrm{g}}$, and to the temperature where the particulate morphology disappears. ${ }^{5}$ The value of $G^{\prime \prime}$ is more difficult to reproduce than $G^{\prime}$; this fact indicates that there is more variation in the void volume than in the structure. These voids are interpreted as the interparticulate spaces observed in the electron micrographs of the gelled, but unfused specimens. ${ }^{5}$

The present work is also directed toward finding a relationship between the viscoelastic response of the 


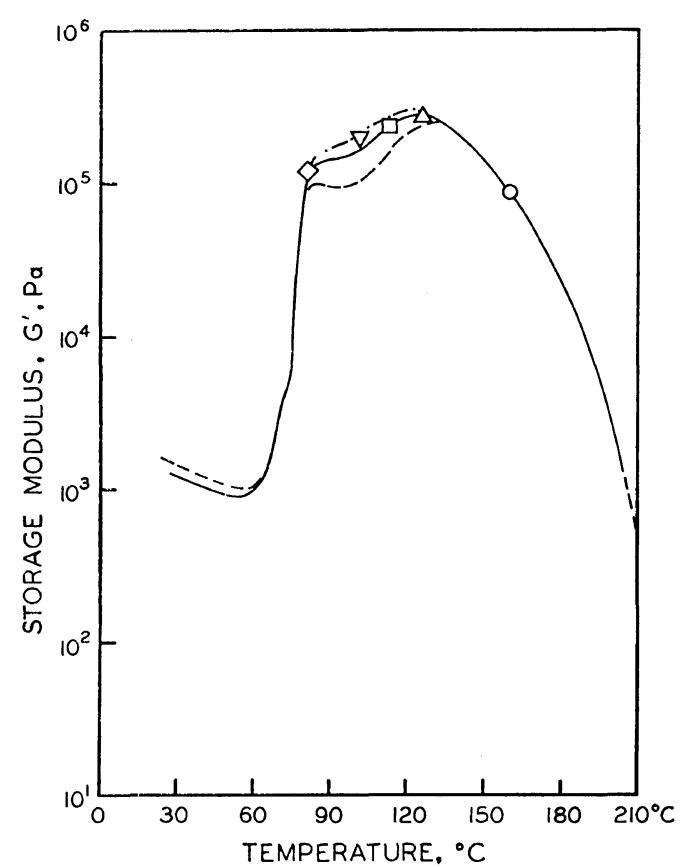

(a)

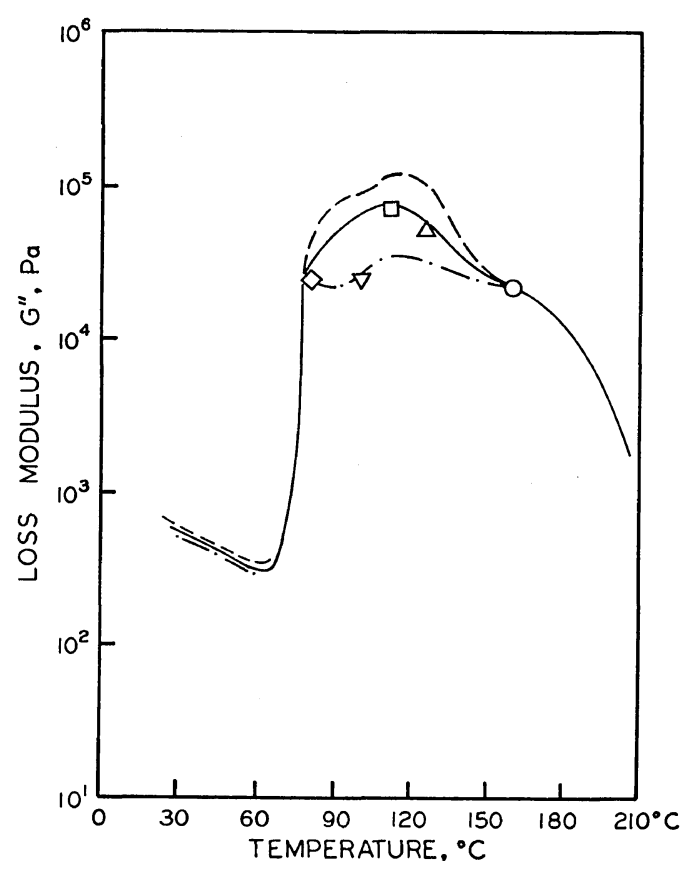

(b)

Figure 3. Viscoelastic profile of gelation and fusion of plastisol made with FF fraction, (a) storage modulus and (b) loss modulus; plastisol aged for more than a year.

Table III. Sampling temperature for morphological study and respective viscoelastic characteristics

\begin{tabular}{|c|c|c|c|}
\hline \multicolumn{3}{|c|}{ Temperature $/{ }^{\circ} \mathrm{C}$} & \multirow{2}{*}{$\begin{array}{l}\text { Viscoelastic } \\
\text { characteristics }\end{array}$} \\
\hline FF & $4 \mathrm{C}$ & RF & \\
\hline 81 & 87 & 88 & 1st peak, past $T_{\mathrm{g}}$ \\
\hline 101 & 96 & 102 & inflection in $G^{\prime}$ \\
\hline 113 & - & - & 2nd $G^{\prime \prime}$ peak \\
\hline 126 & 129 & 129 & 2nd $G^{\prime}$ peak \\
\hline 160 & 159 & 154 & Fusion becoming dominant \\
\hline 210 & 210 & 210 & Fusion complete \\
\hline
\end{tabular}

fusing plastisol and the corresponding morphological structure. The temperatures at which the morphological specimens were taken are indicated by the markings in Figure 3 and are listed in Table III.

Figures 4 and 5 likewise show the viscoelastic data taken during the gelation and fusion processes. Again, there were difficulties in reproducing the $G^{\prime \prime}$ data over the same temperature range. However, the scatter in the data is somewhat less than that observed for the FF fraction. The $G^{\prime}$ data were reproduced satisfactorily. These facts reinforce the speculation that there is variation in the void volume. The source of the scatter in the data is neither the nonuniformity in molecular weight nor the resin-plasticizer concentration, since these variations would have been observed in the fusion data at temperatures above $160^{\circ} \mathrm{C}$. The lack of reproducibility cannot be attributed to nonuniformity in the particle size distribution either, because this would have been apparent in the data below $55^{\circ} \mathrm{C}$, where the specimen consisted of PVC particles suspended in a continuous phase.

The fact that the most non-reproducible data was encountered in experiments using the plastisols of the FF fraction may be attributed to the following cause. The FF fraction consisted as a first approximation of only fine particles, and so its plastisol was very high in viscosity and very thixotropic. Therefore, the sample loading history might have imparted a variable thixotropic memory, which is perhaps magnified once gelation occurs. This hypothesis was tested by the following procedure: a sample was loaded into the Rheometrics Mechanical Spectrometer and the plattens were closed. In this state, the sample was left for two hours at 
Gelation and Fusion of PVC Plastisol

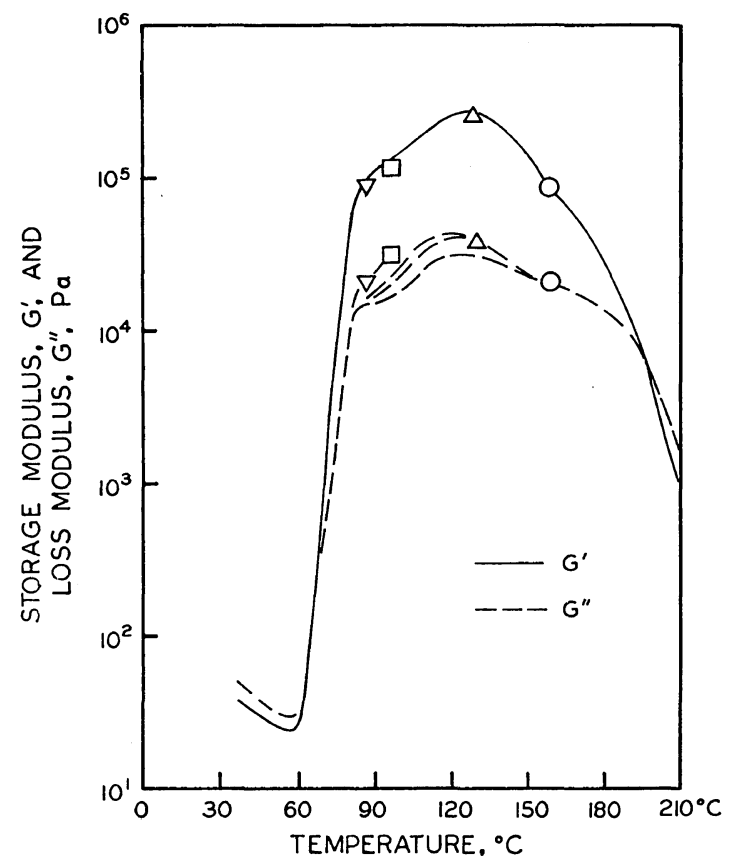

Figure 4. Viscoelastic profile of gelation and fusion of plastisol made with $4 \mathrm{C}$ fraction; plastisol stored for more than a year.

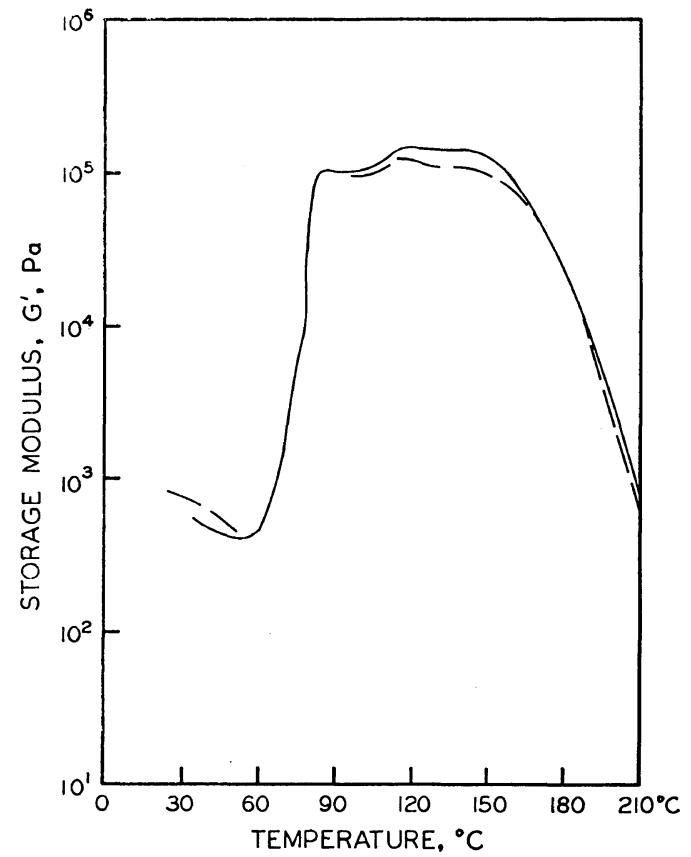

(a)

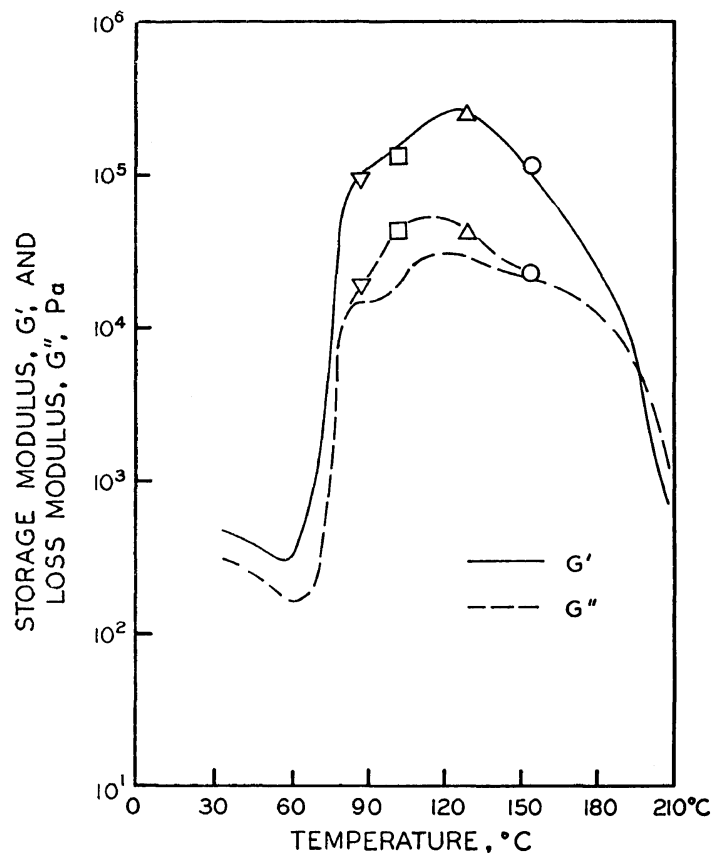

Figure 5. Viscoelastic profile of gelation and fusion of plastisol made with RF sample; plastisol stored for more than a year.

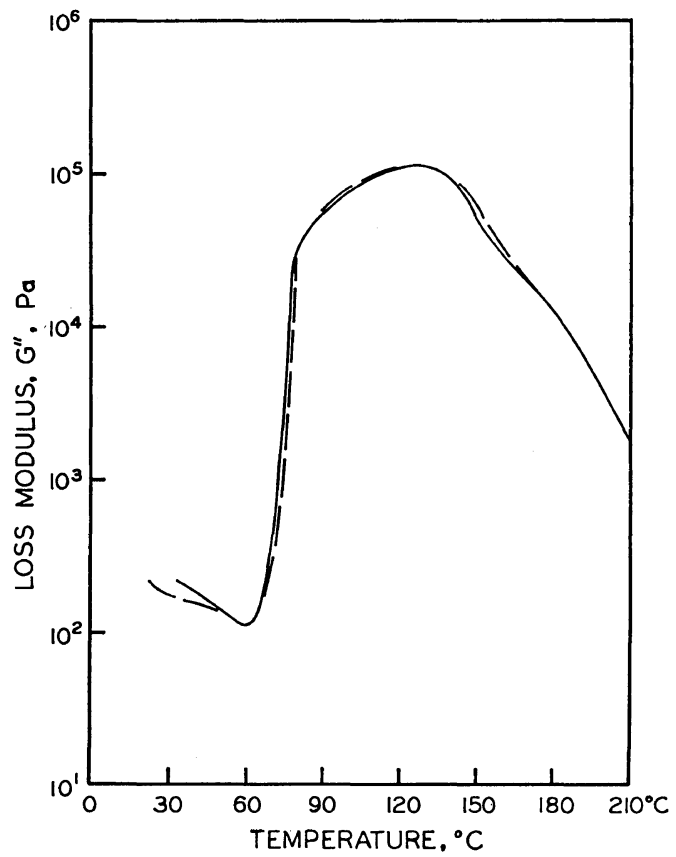

(b)

Figure 6. Viscoelastic profile of gelation and fusion of plastisol made with FF fraction, (a) storage modulus and (b) loss modulus; newly prepared plastisol. 
N. NAKAJIMA et al.

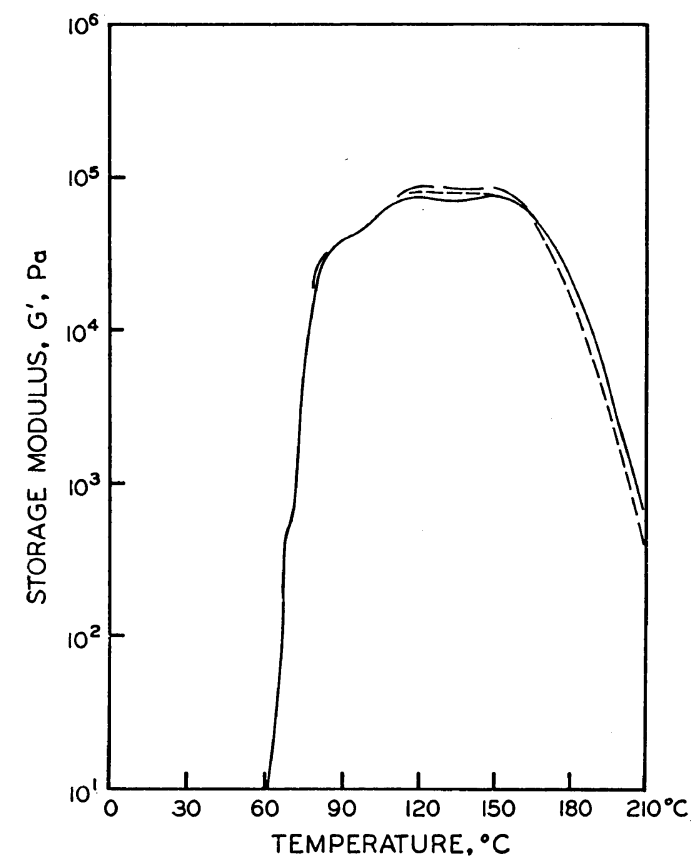

(a)

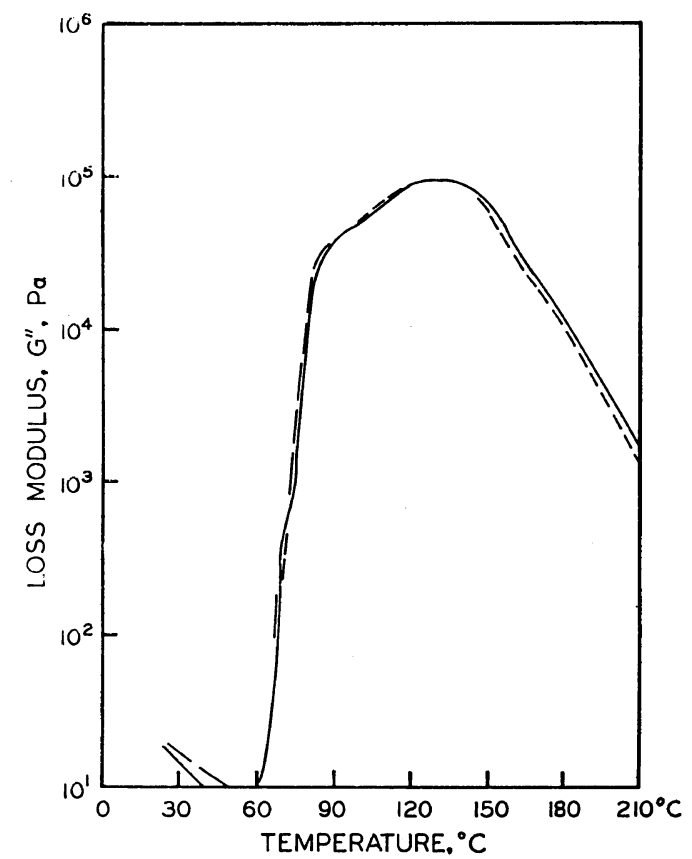

(b)

Figure 7. Viscoelastic profile of gelation and fusion of plastisol made with 4C fraction, (a) storage modulus and (b) loss modulus; newly prepared plastisol.

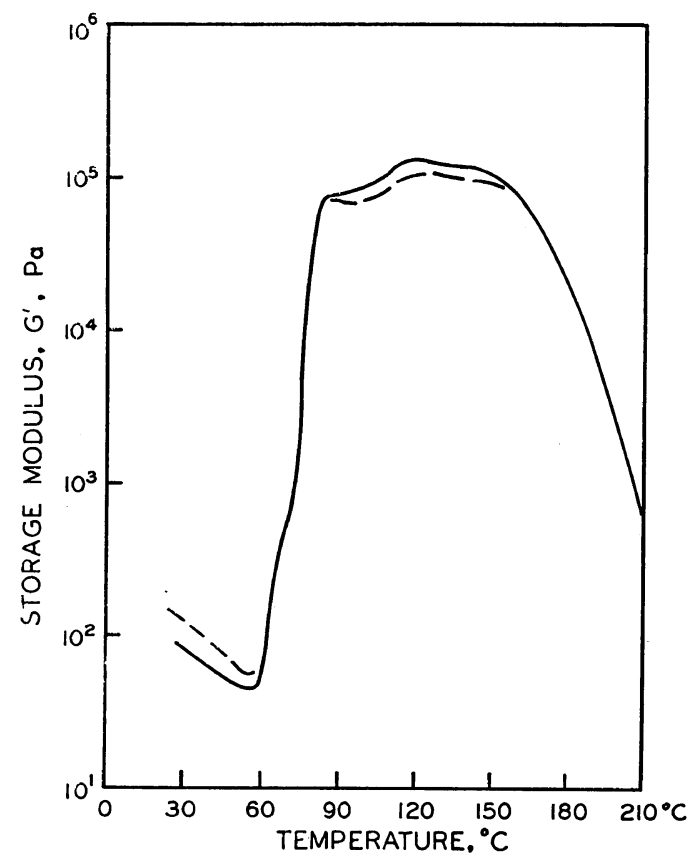

(a)

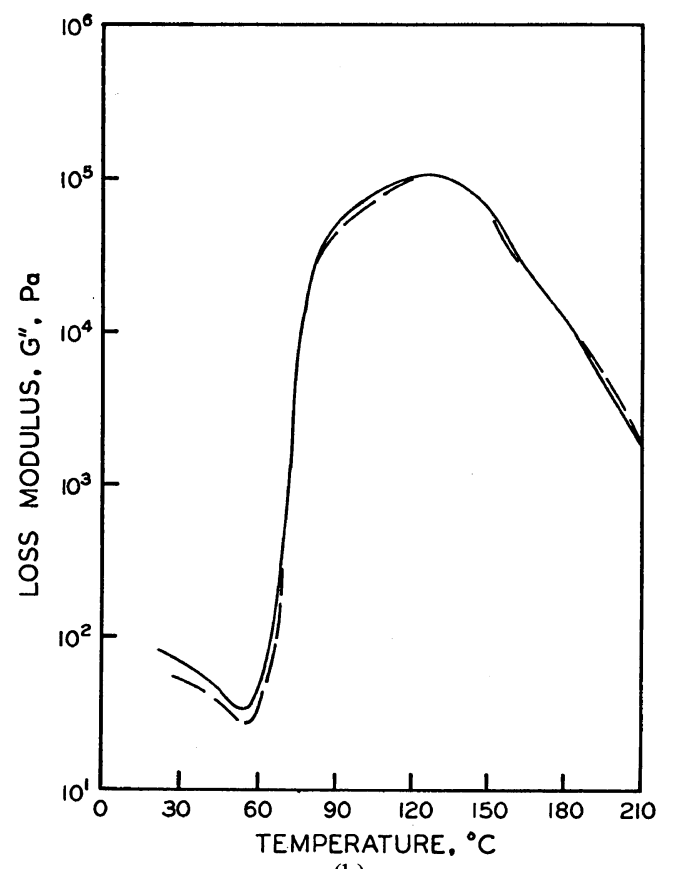

(b)

Figure 8. Viscoelastic profile of gelation and fusion of plastisol made with $\mathrm{FF} / 4 \mathrm{C}, 50 / 50$ blend, (a) storage modulus and (b) loss modulus; newly prepared plastisol. 


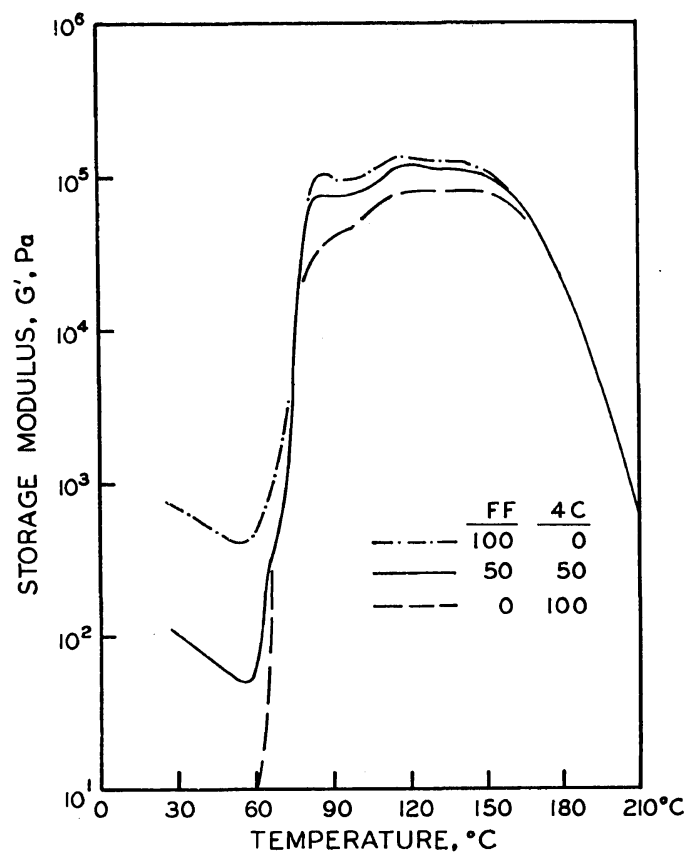

(a)

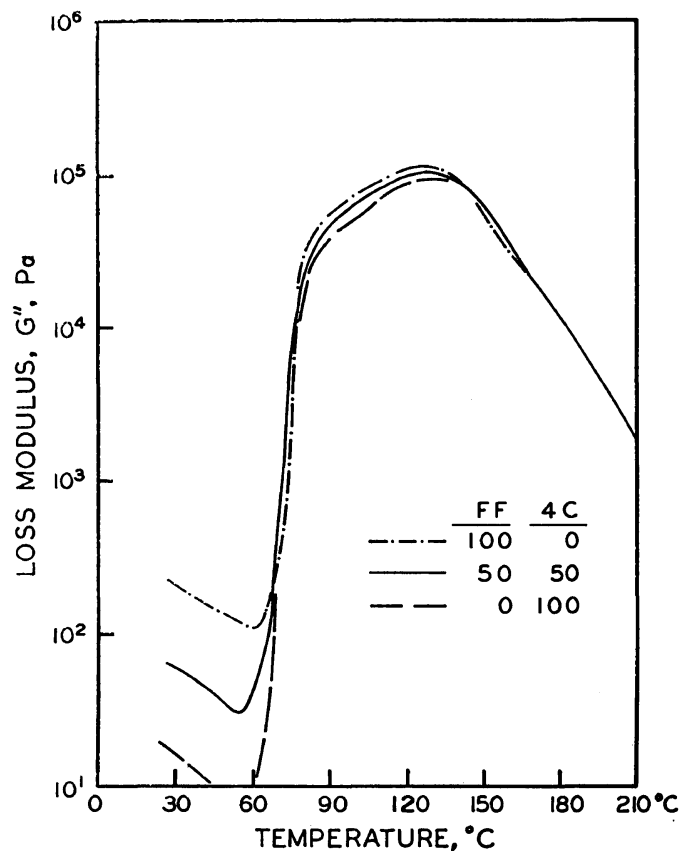

(b)

Figure 9. Effect of particle size distribution on the viscoelastic profile of gelation and fusion, (a) storage modulus and (b) loss modulus.

room temperature in order to erase the memory of loading. Then, the viscoelastic measurement was performed for characterizing the gelation and fusion. The results will be discussed later.

Another source of error may be attributed to the aging of the plastisols. The plastisol mixes were more than a year old, and fresh plastisols were prepared from the same resin fractions.

Figures 6, 7, and 8 show the data for newly prepared plastisols. Since there was a significant increase in viscosity in the first two or three weeks, the plastisols were kept for about a month to allow the viscosity to stabilize. The reproducibility of the data was quite satisfactory with the new series of plastisols, as well as with other blends, $\mathrm{FF} / 4 \mathrm{C}$ ratios of $85 / 15,75 / 25$ and $25 / 75$. With all the samples the measurements were carried out at least in duplicate, one being immediately and the other, $2 \mathrm{~h}$ after loading. These two measurements were made after a sufficient aging period, and about 14 days apart. With these new plastisols, the loading memory did not affect the reproducibility of the data.

The effect of particle size distribution (PSD) is shown in Figure 9, where the gelation and fusion profiles of FF, 4C and their 50-50 blend are com-

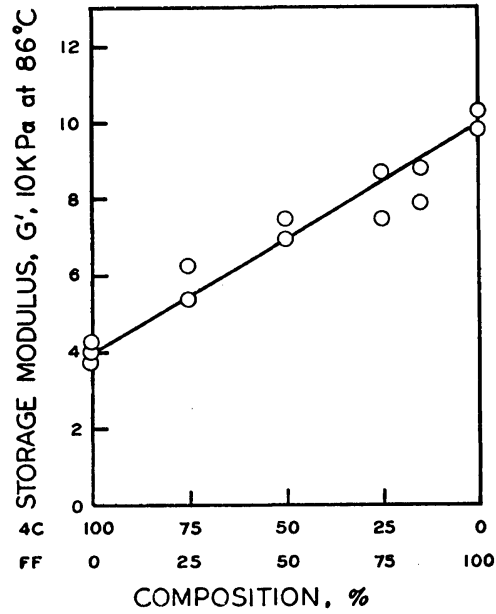

Figure 10. Effect of particle size distribution on storage modulus at $86^{\circ} \mathrm{C}$.

pared. The greatest differences are seen in the temperature range where the plastisol exists in a suspension state, and where the fine particles increase both $G^{\prime}$ and $G^{\prime \prime}$. ${ }^{2}$ These differences were carried over to the beginning of gelation. The effects of PSD during gelation and fusion are much smal- 
ler, appearing in the temperature range between $T_{\mathrm{g}}$ and $160^{\circ} \mathrm{C}$, where the particulate structure and interparticle voids are present.

Figure 10 is the $G^{\prime}$ data for all blends and components at $86^{\circ} \mathrm{C}$, which is a few degrees above $T_{\mathrm{g}}$. A trend is clearly shown that the finer particles give a higher $G^{\prime}$. The same trend exists with $G^{\prime \prime}$, but the effect is much smaller.

The fact that the fine particles gave the higher $G^{\prime}$ indicates that the fine particles enhance the formation of interparticle bonding. ${ }^{5}$ The higher $G^{\prime \prime}$ resulting from the presence of fine particles implies a larger amount of void volume. ${ }^{5}$

\section{MORPHOLOGY}

The SEM photographs of plastisol made with FF fraction are shown in Figures 11, 12, and 13 for all sampling temperatures (Table III) except for $113^{\circ} \mathrm{C}$. The photograph for the $113^{\circ} \mathrm{C}$ sample is not shown, because there was no sufficient change in morphology to yield significant information. Figure 12 also shows the morphology during gelation, 96$102^{\circ} \mathrm{C}$ of samples $4 \mathrm{C}$ and RF. Figure 13 shows the morphology during the fusion $154-160^{\circ} \mathrm{C}$, of the three samples. The SEM photographs of $4 \mathrm{C}$ and $\mathrm{RF}$ samples at other temperatures are similar to those of the FF sample at comparable temperatures and thus they are not reproduced here.

There are several common features among the three samples: (i) the particulate structure and the interparticulate voids are clearly visible in the temperature range from 81 to $129^{\circ} \mathrm{C}$. (ii) The photographs of samples removed from the RMS at $81-$ $88^{\circ} \mathrm{C}$ show particles wet with DOP. (iii) in the $81-$ $129^{\circ} \mathrm{C}$ range, a gradual development of the interparticle bonding is indicated. (iv) The particles observed in the photographs are primary particles, i.e., the particles produced in polymerization. The size of the swollen particles, for example, at 126$129^{\circ} \mathrm{C}$, is about $1 \mu \mathrm{m}$ or smaller. This is in good agreement with the size of the fine particle resin powder, which had a median diameter of $0.65-$ $0.73 \mu \mathrm{m}^{2}{ }^{2}$ The corresponding median size of the swollen particle is $0.80-0.90 \mu \mathrm{m}$. (v) At 154$160^{\circ} \mathrm{C}$ the fusion is almost complete and no particulate structure is visible. However, the fracture surface indicates a domain boundary of several tens of micrometers. Evidently, many defects are present, leading to the crack-propagation exhibited in

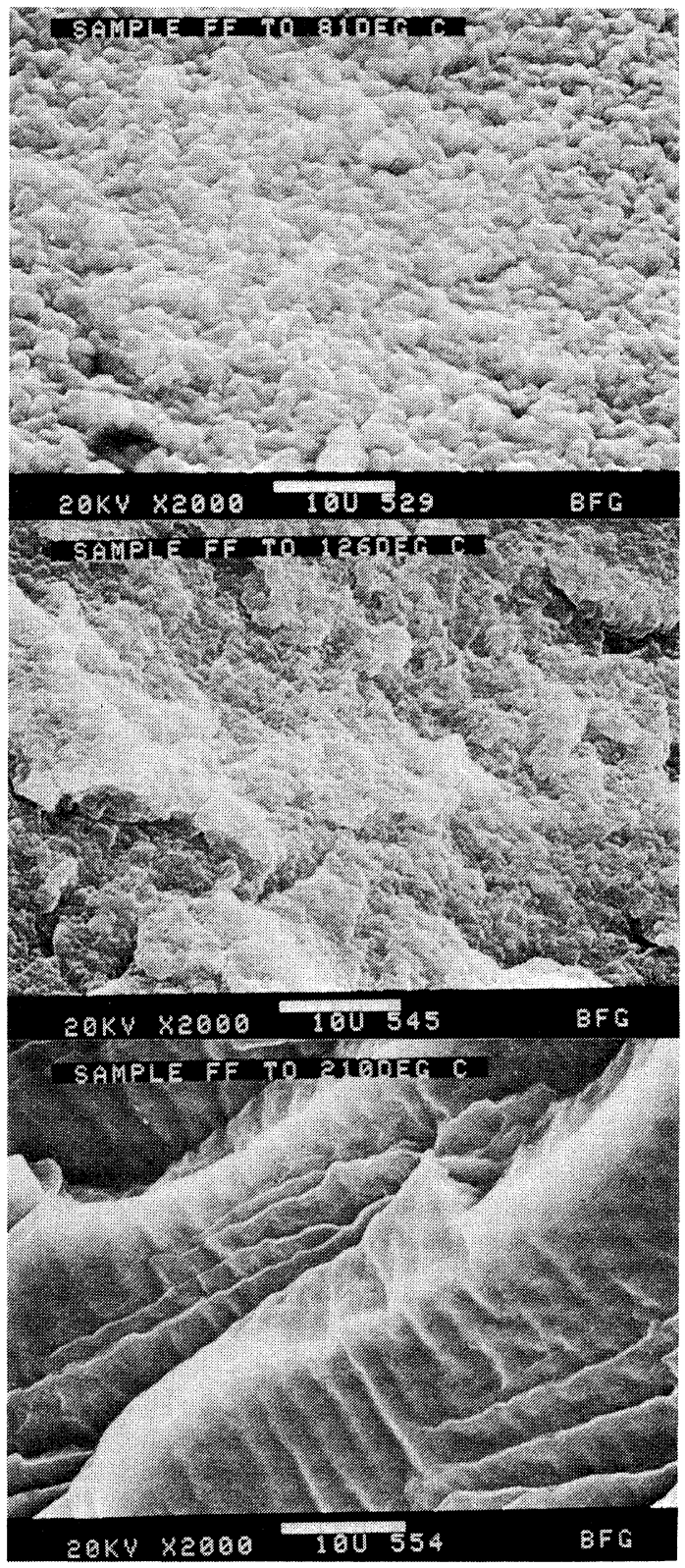

Figure 11. Changes in morphology with progress of gelation and fusion. $2000 \times$

the photographs. (vi) At $210^{\circ} \mathrm{C}$ the fusion is complete. The fracture surface is more like that of a tough, glassy material. The manner of crack propagation indicates that there are significantly fewer defects.

A major compositional difference among the FF, 


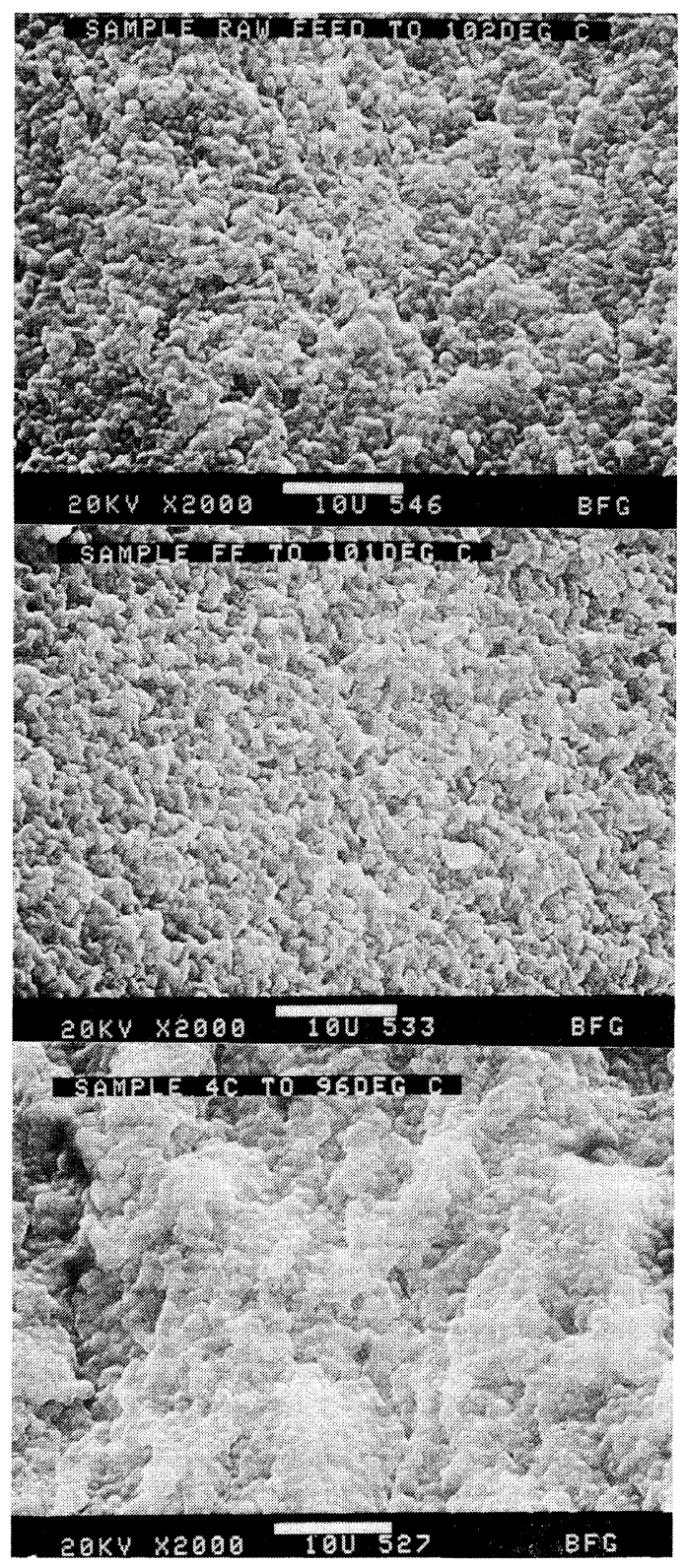

Figure 12. The effect of particle size distribution on morphology during gelation. $2000 \times$

4C, and RF samples is the amount of the coarse particles about $15 \mu \mathrm{m}$ in the median diameter. These particles are agglomerates formed during the spray drying. The presence of such agglomerates is inferred in the photographs of $4 \mathrm{C}$ specimens which were heated to $96^{\circ} \mathrm{C}$, Figure 12 . However, very similar fracture surfaces are seen for the FF and RF

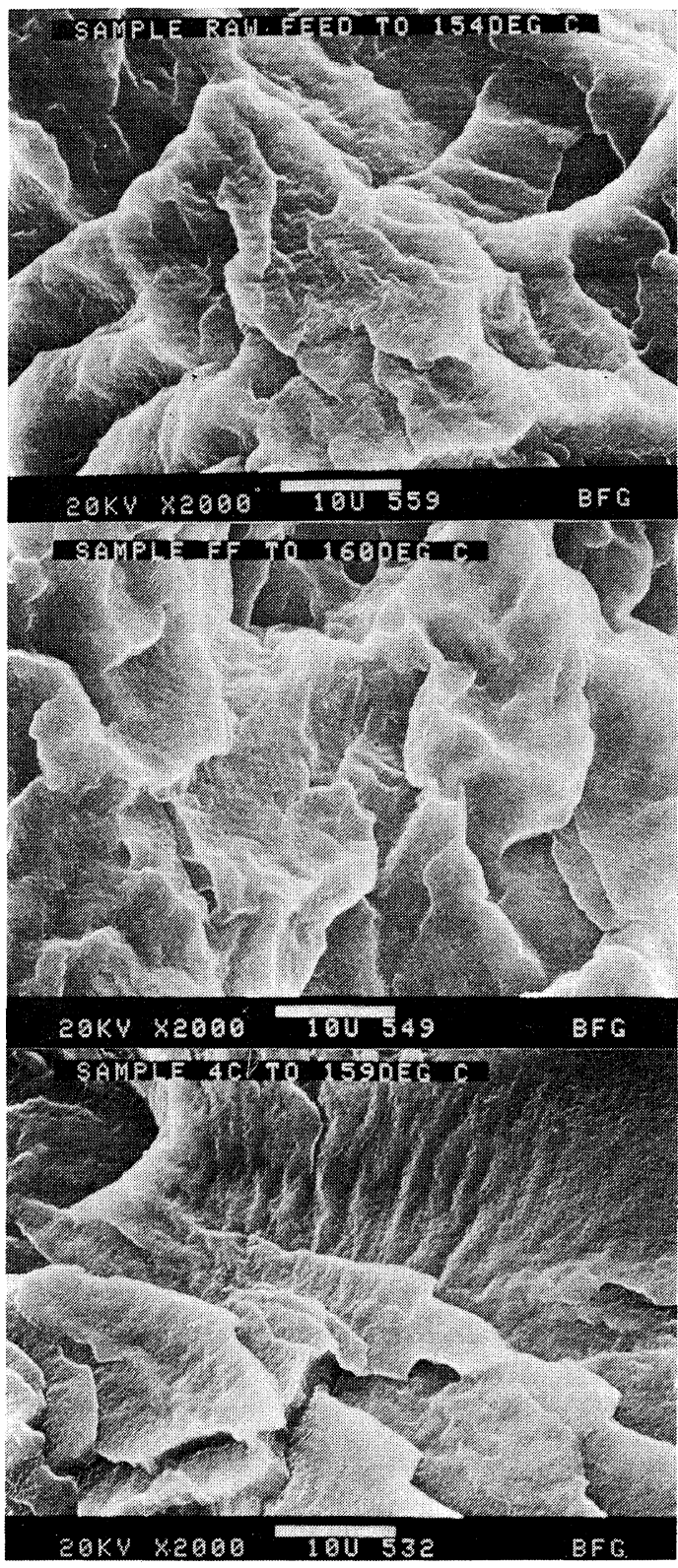

Figure 13. Absence of the effect of particle size distribution on morphology during fusion. $2000 \times$

samples. The RF sample contains only $18 \%$ agglomerated particles which the SEM perhaps failed to spot. There is no noticeable difference in the fracture surfaces of three samples during fusion, (Figure 13). The effect of particle size distribution is no longer apparent, because the particulate structure has disappeared. 


\section{N. NAKAJIMA et al.}

\section{DISCUSSION}

The extent to which particle size distribution of PVC plays a role in gelation and fusion behavior has been characterized by the described viscoelastic measurements. This was done using plastisols prepared and aged for several weeks. The plastisol samples which were more than a year old gave data difficult to reproduce in the exact temperature range where the effect of particle size distribution would have been observable. The plasticization at room temperature of very fine particles seems to have taken place during this long period of aging. However, how this could affect the variability of the results cannot be explained quantitatively.

With the newly prepared plastisols and after several weeks of aging, the viscoelastic data were very reproducible even though the duplicate measurements were made a few weeks apart. The effect of particle size distribution is that the larger amount of fine particles increases both $G^{\prime}$ and $G^{\prime \prime}$ over the temperature range between 81 and $155^{\circ} \mathrm{C}$. This trend is more pronounced with $G^{\prime}$ and somewhat less with $G^{\prime \prime}$.

When a greater amount of fine particles is present, there is a smaller amount of coarse particles. Therefore, it is still open to question as to whether the coarse or fine particles are responsible for the observed differences in behavior. However, the fine particles are most probably responsible for the following reason; the moduli range between 81 and $155^{\circ} \mathrm{C}$ is $10^{4}$ to $10^{5} \mathrm{~Pa}$. These moduli correspond to those of polymer melts and unfilled elastomers which contain a network structure, normally called "entanglements." The effect of having larger particles in these systems is like having a filler which normally increases the moduli, instead of decreasing them. On the other hand, with a larger amount of fine particles there is the probability of a larger amount of interparticle contacts per unit mass of polymer. Consequently, the system behaves as if it had a larger number of the "network" junctions, accounting for the higher $G^{\prime}$. Likewise, with more fine particles there is a larger number of the interparticle voids, which contribute to the enhanced loss mechanism. Hence, this results in a somewhat higher $G^{\prime \prime}$.

The above mechanism, although plausible, can not entirely be supported by morphological observations. That is, the large differences in the amount of fine particles in the original plastisols, (Table II), are not clearly discernible in the fractured surfaces. Instead, the coarse particles might have been somewhat deagglomerated. This, of course, diminishes sample-to-sample differences with respect to the amount of the fine particles.

The viscoelastic measurements in this investigation were performed at $1 \mathrm{rad} \mathrm{s}^{-1}$. In commercial processes, the deformation rate is usually much slower. An estimated deformation rate of the foam process is 0.1 to $0.01 \mathrm{~s}^{-1}$. Other processes such as slush-molding and dip-coating are done at even lower rates. The differences in both the density of the "network" junctions and the amount of voids (will) results in increasingly greater differences in viscoelastic data at slower deformation rates. Therefore, the results of this study are of significant practical interest, since it is expected that even larger differences with higher dependence on particle size distribution will become apparent in certain commercial processes.

\section{CONCLUSION}

The effect of particle size distribution of PVC dispersion resins on the gelation and fusion behavior of their plastisols can be characterized by viscoelastic measurements. The dynamic moduli at the intermediate stage of gelation-fusion process is affected by the particle size distribution. The morphological observation of the fracture surfaces clearly shows that at this stage the system has a particulate structure. The larger fraction of fine particles gives higher values of $G^{\prime}$ and $G^{\prime \prime}$. However, it has not been resolved yet as to whether the fraction of fine in general or the fraction of ultrafine is responsible. A plausible cause of the observed effect is that the fine or ultrafine particles give more interparticle bonding, hence, the higher $G^{\prime}$ and more void volume, hence the higher $G^{\prime \prime}$.

Acknowledgements. The authors wish to express their gratitude to the BFGoodrich Chemical Group for permission to publish this work. The morphological specimens were prepared and examined by R. G. Raike, the particle size distributions were measured by $H$. V. Flint and the viscoelastic measurements were made by $\mathrm{C}$. W. Woods. 


\section{REFERENCES}

1. E. A. Collins, D. J. Hoffmann, and P. L. Soni, Rubber Chem. Technol., Rubber Review, 52, 676 (1979).

2. N. Nakajima and C. A. Daniels, J. Appl. Polym. Sci., 25, 2019 (1980).

3. N. Nakajima, D. W. Ward, and E. A. Collins, Polym. Eng. Sci., 19, 210 (1979).

4. N. Nakajima and D. W. Ward, a paper presented at 52nd Annual Meeting of The Society of Rheology,
Williamsburg, Virginia, February 23-25, 1981.

5. N. Nakajima, J. D. Isner, and E. R. Harrell, a paper presented at 3rd International Symposium on Poly(vinyl chloride), Cleveland, Ohio, August 1015, 1980; J. Macromol. Sci. Phys., in press.

6. Micromertics Instrument Company, Norcross, Georgia.

7. E. A. Collins, J. A. Davidson, and C. A. Daniels, $J$. Paint Technol., 47, 45 (1975).

8. Rheometrics, Inc., Union, New Jersey.

9. AMRAY, Inc., Bedford, Massachusetts, 01730. 